\title{
SMURFS: Superpixels from Multi-scale Refinement of Super-regions
}

Imanol Luengo ${ }^{1}$

imanol.luengo@nottingham.ac.uk

Mark Basham ${ }^{2}$

mark.basham@diamond.ac.uk

Andrew P. French ${ }^{1}$

andrew.p.french@nottingham.ac.uk
${ }^{1}$ School of Computer Science, University of Nottingham, Nottingham, UK, NG8 1BB

${ }^{2}$ Diamond Light Source Ltd, Harwell Science \& Innovation Campus, Didcot, UK, OX11 ODE

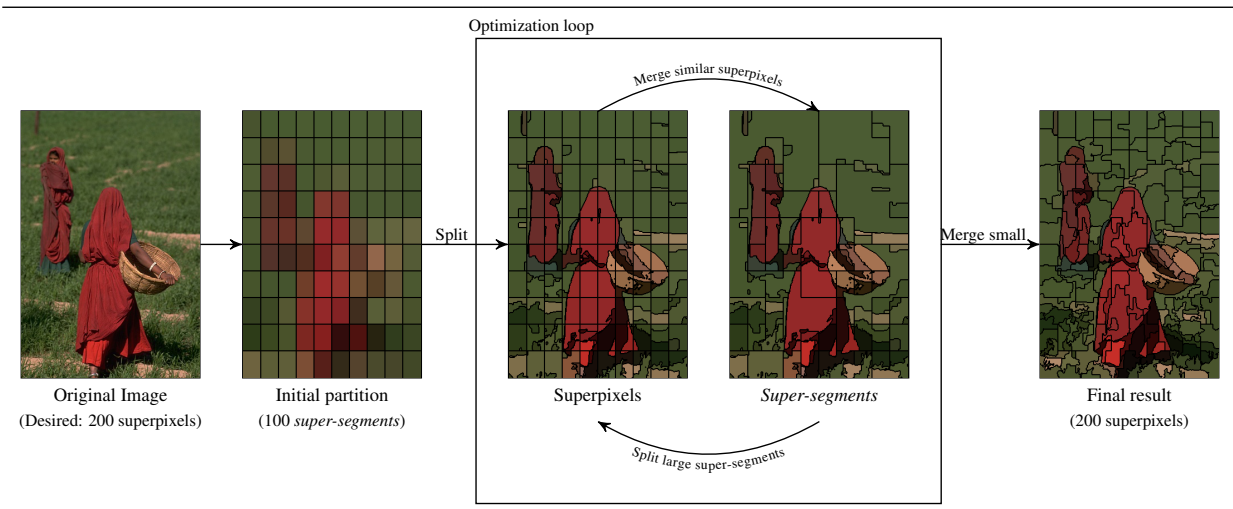

Figure 1: Overview of our algorithm. Iterative refinement over two scales of regions yields increasingly more robust superpixels that better capture global image features.

Here we present a new superpixel algorithm: Superpixels from MUlti-scale ReFinement of Super-regions (SMURFS), which not only obtains state of the art superpixels, but can also be applied hierarchically to form what we call $n$ th order super-regions. In essence, starting from a uniformly distributed set of super-regions, the algorithm iteratively alternates graph-based split and merge optimization schemes which yield superpixels (1st order super-regions) that better represent the image. We define a super-region hierarchy forming the level $i$ by grouping elements of the level $i-1$. Denoting the pixel grid as level $i=0$, superpixels (level $i=1$ ) are formed by grouping similar adjacent pixels while supersegments (level $i=2$ ) are formed of multiple superpixels. To be able to better represent the image, we alternate optimization schemes at both level $i=1$ and $i=2$ with the aim of refining both superpixels and supersegments simultaneusly. The split step is performed over the pixel grid to separate large supersegments into different smaller superpixels. This step is fully parallelizable as every region is split independently, and produces superpixels that better capture local information of the supersegments. The merging process, conversely, is performed over the superpixel graph to create supersegments with the aim of better capturing global image features. This iterative twoscale procedure refines the super-region boundaries of the image without shape or boundary initialization constraints, present in most of state of the art superpixels. Results show state of the art Achievable Segmentation Accuracy (ASA) in the Berkley Segmentation dataset (BSD500) [1].

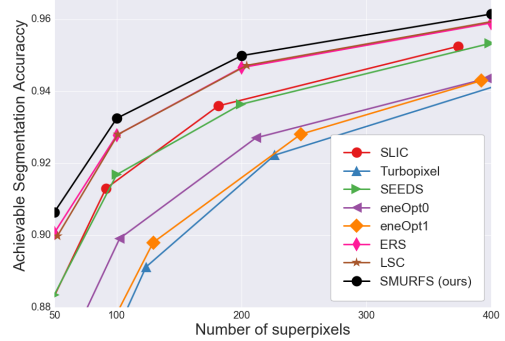

Figure 2: ASA comparison on the BSD500 dataset.

[1] D. Martin, C. Fowlkes, D. Tal, and J. Malik. A database of human segmented natural images and its application to evaluating segmentation algorithms and measuring ecological statistics. In Proc. 8th Int'l Conf. Computer Vision, volume 2, pages 416-423, July 2001. 